\title{
Connector Enhancer of Kinase Suppressor of Ras 1
}

National Cancer Institute

\section{Source}

National Cancer Institute. Connector Enhancer of Kinase Suppressor of Ras 1. NCI

Thesaurus. Code C25990.

Connector enhancer of kinase suppressor of ras 1 (720 aa, $\sim 80 \mathrm{kDa}$ ) is encoded by the human CNKSR1 gene. This protein plays a role in the modulation of protein-protein interactions during Ras signaling. 